\title{
Collimation technique and testing applied to finite size polychromatic sources
}

\author{
Francisco Jose Torcal-Milla ${ }^{1, *}$ and Luis Miguel Sanchez-Brea ${ }^{2}$ \\ ${ }^{1}$ Universidad de Zaragoza, Departamento de Física Aplicada, 50009, Zaragoza (Spain) \\ ${ }^{2}$ Universidad Complutense de Madrid, Facultad de Ciencias Fisicas, Departamento de Óptica, Plaza de las Ciencias 1, 28040, Madrid (Spain) \\ *Corresponding author: fjtorcal@unizar.es
}

Compiled March 17, 2017

\begin{abstract}
Highly collimated beams are required in numerous applications and techniques. Different methods have been proposed for collimating monochromatic point light sources during the recent years. In this work, we analyze how a finite size and polychromatic light source can be collimated using only one diffraction grating and a CMOS camera placed after the source and the collimating lens. For this, we determine the period of the fringes diffracted by the grating and compare it with the period of the grating. Analytical equations are obtained to predict the amplitude of the fringes and their period. Since selfimages disappear for finite size polychromatic sources at long distances from the grating, the period has to be measured close to the grating. In addition, we give analytical equation to determine the error in the positioning of the source in terms of the source size and the set-up parameters. Finally, we experimentally corroborate the obtained analytical formalism using a white LED of size $s=0.6 \mathrm{~mm}$ collimated by a lens with focal length $f=25 \mathrm{~mm}$, and a Ronchi binary grating of period $d=250 \mu \mathrm{m}$. In this case, we achieve an experimental error in the positioning of the source with respect to the focal plane of the lens of $\delta z_{\text {exp }}=92 \mu \mathrm{m}$. ( 2017 Optical Society of America
\end{abstract}

OCIS codes: $\quad$ (050.1950) Diffraction gratings(140.3490); (070.6760) Talbot and self-imaging effects; (120.1680) Collimation.

http://dx.doi.org/10.1364/ao.XX.XXXXXX

\section{INTRODUCTION}

Theoretically, a beam is collimated when it does not change neither its shape nor its size when it propagates along space. Lamentably, this definition is highly restrictive and it can be applied only when the light source is a monochromatic point-like source. When this is not the case, the definition of collimation must be slightly relaxed and we need to talk about the "higher possible collimation degree of the beam". We define the collimation degree as the lesser deviation of the rays forming the beam. Lesser deviation results in higher collimation degree. Collimated beams are crucial for many applications and techniques, scientific and technological. It is a very important technique in areas such as holography, information processing, metrology, lithography, instrumentation, illumination, satellites alignment, free space communications, etc. In general, the most simple technique is the so-called auto-collimation technique. It consists of measuring the size of the beam at two different distances and comparing both measures [1]. Although, this technique is quite imprecise due to the proper propagation of the beam that modifies its shape in some way.

Other more precise techniques based on different optical effects have been developed along the years [2-10]. These techniques improve the collimation degree of the beams in several orders of magnitude. Collimation methods based on Talbot interferometry use Talbot effect to create a moiré pattern at the output of a double grating system where the second grating is placed at a self-image of the first one [1, 11-14]. This idea has been modified in several ways in the recent years: by lateral displacement of one of the gratings, by changing the periods of the gratings, by changing their shape, etc [15-17]. Another simple procedure to evaluate the collimation degree of a light beam consists of using the Lissajous figure produced by a double diffractive optical system formed by a diffraction grating and a more complex mask with two diffraction gratings of the same period as the first grating but laterally shifted [18]. With this technique, a continuous movement of the grating is required to determine the collimation degree of the beam. This fact is solved in [19] where the set-up is modified by changing the mask by a more complex one. Thus, the collimation state of the beam is directly measured without moving any element of the set-up. Another technique consists of replacing one of the linear gratings by a circular grating [17], resulting in a method that does not need either any 
displacement of the optical elements to check the collimation degree of the beam. Unfortunately, the collimation degree is obtained by means of a complex analysis of the parabolic fringes formed after the second grating, [20]. This advantage is also found in a recently developed method based on measuring the demagnification/magnification of the period of the selfimages produced by a diffraction grating after the collimating lens [21]. In this case, only one diffraction grating is needed. Summarizing, when the beam passes through the diffraction grating, self-images with maximum contrast are located at multiples of the Talbot distance, $z_{T}=2 l d^{2} / \lambda$, where $d$ is the period of the grating, $l$ is an integer, and $\lambda$ is the illumination wavelength [22]. The beam is collimated when the period of the grating, $d$, and the period of the self-image at the sensor, $d^{\prime}$, are equal, since light after the grating propagates parallel to the propagation axis that is perpendicular to the grating. Some studies of collimation techniques for polychromatic sources have been performed in [23-25], but most of the previous works have been developed for monochromatic point light sources where self-images are commonly studied. On the other side, self-imaging of finite size polychromatic sources has been exhaustively analyzed in [26]. The authors show how polychromatic light produces a kind of continuous self-imaging regime without the contrast inversion inherent to the Talbot effect. Besides, finite size of the source produces a gradual disappearance of the fringes for longer distances, lowering its contrast.

In this work, we redefine the collimation technique shown in [21] for the case of a polychromatic and finite size light source instead of a monochromatic point source. The analysis becomes more complex but analytical equations can also be obtained. With them, we can predict the maximum distance where fringes with high contrast can be obtained and the corresponding error in the positioning of the source in terms of the source size and the remaining set-up parameters. Collimation testing is achieved by comparing the period of the diffraction fringes with the period of the grating. Due to the finite size of the source, and according to [26], the presented technique must be applied to the zeroth or first self-image to obtain enough contrast of the fringes to be measured accurately. In addition, the period dependence on the distance from the grating becomes parabolic and additional considerations must be taken into account, in comparison to monochromatic pointlike light source.

\section{BEAM COLLIMATION TECHNIQUE AND TESTING: ANALYTICAL APPROACH}

Let us consider the scheme depicted in Fig. 1. Firstly, we consider a point and monochromatic incident light source placed at a distance $x_{0}$ from the optical axis, whose wavelength is $\lambda$, that illuminates a system formed by a lens $L$ whose focal length is $f$, an amplitude diffraction grating $G$ of period $d$, and a CMOS camera. The distance from the source to the lens $L$ is $z_{0}$, from the lens $L$ to the grating $G$ is $z_{1}$, and from $G$ to the camera is $z_{2}$, respectively. After calculations, we will extend our analysis to polychromatic finite extension light sources by performing two integrals over the size of the source and the wavelength interval around the central wavelength.

Considering a point light source placed at a distance $z_{0}$ from a lens and $x_{0}$ from the optical axis, the field at a distance $z_{1}$ after

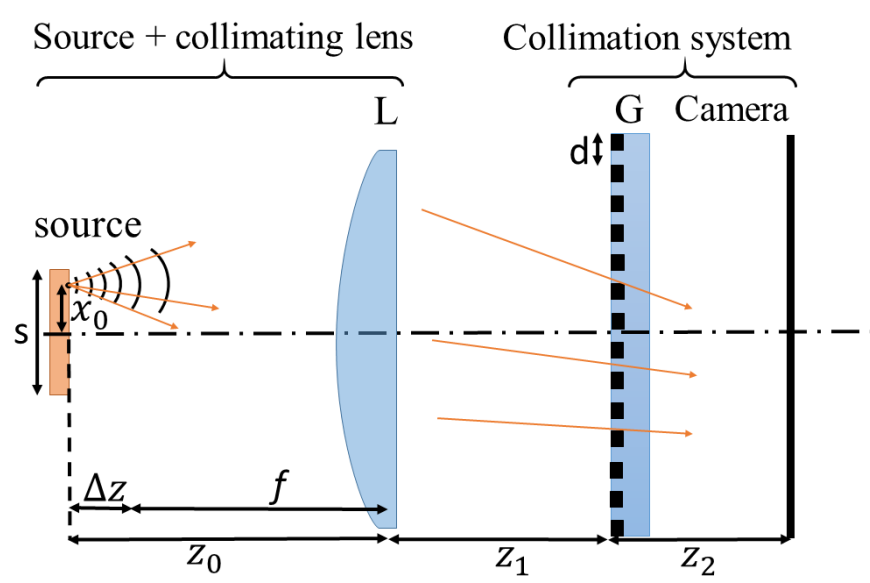

Fig. 1. Scheme of the set-up proposed for collimating the finite size polychromatic source showing the parameters involved. $L$ is the collimating lens and $G$ is the diffraction grating.

the lens in paraxial approximation can be expressed as $[18,21]$,

$$
U_{1}\left(x_{1}\right)=U_{0} e^{\frac{i k f}{2} \frac{\left(x_{0}-x_{1}\right)^{2}}{f\left(z_{0}+z_{1}\right)-z_{0} z_{1}}} e^{-\frac{i k}{2} \frac{\left(x_{1}^{2} z_{0}+x_{0}^{2} z_{1}\right)}{f\left(z_{0}+z_{1}\right)-z_{0} z_{1}}}
$$

where $U_{0}$ is the amplitude of the incident beam, $x_{1}$ is the transversal position after the lens, $k=2 \pi / \lambda$, and $i=$ $\sqrt{-1}$ is the imaginary unit. After, the light beam passes through the diffraction grating, which is characterized by its Fourier series expansion, $t(x)=\sum_{n=-\infty}^{\infty} a_{n} \exp ($ inq $x)$, where $a_{n}$ are the Fourier coefficients of the grating, $q=$ $2 \pi / d$, and $n$ are integers. The field after the grating considering thin element approximation is then $U_{1}^{\prime}\left(x_{1}\right)=$ $U_{1}\left(x_{1}\right) t\left(x_{1}\right)$. To obtain the field at a distance $z_{2}$ from the grating we use again the Fresnel approach, $U_{2}\left(x_{2}\right)=$ $\left(e^{i k z} / i \lambda z_{2}\right) \int U_{1}\left(x_{1}\right) \exp \left[i \frac{k}{2 z_{2}}\left(x_{1}-x_{2}\right)^{2}\right] d x_{1}$, resulting in

$$
\begin{aligned}
U_{2}\left(x_{2}, z_{2}\right)= & C_{2} \sum_{n=-\infty}^{\infty} a_{n} e^{-i \frac{n^{2} q^{2} z_{2}}{2 k\left\{1+\left(f-z_{0}\right) z_{2} /\left[-z_{0} z_{1}+f\left(z_{0}+z_{1}\right)\right]\right\}}} \\
& \times e^{i \frac{n q x_{0}}{1+\left[f\left(z_{0}+z_{1}\right)-z_{0}\left(z_{1}+z_{2}\right)\right] /\left(f z_{2}\right)}} e^{i \frac{n q x_{2}}{1+\left(f-z_{0}\right) z_{2} /\left[f\left(z_{0}+z_{1}\right)-z_{0} z_{1}\right]}} \\
& \times e^{i \frac{i\left[f\left(x_{0}-x_{2}\right)^{2}-x_{0}^{2}\left(z_{1}+z_{2}\right)-x_{2}^{2} z_{0}\right]}{2\left[f\left(z_{0}+z_{1}+z_{2}\right)-z_{0}\left(z_{1}+z_{2}\right)\right]}},
\end{aligned}
$$

where $C_{2}$ has taken all constants. Then, the intensity at the observation plane, $I_{2}\left(x_{2}, z_{2}\right)=U_{2}\left(x_{2}, z_{2}\right) U_{2}^{*}\left(x_{2}, z_{2}\right)$, results in

$$
\begin{aligned}
& I_{2}\left(x_{2}, z_{2}\right)=C_{3} \sum_{n, n^{\prime}=-\infty}^{\infty} a_{n} a_{n^{\prime}}^{*} e^{-i \frac{\left(n-n^{\prime}\right) q x_{0}}{1+\left[f\left(z_{0}+z_{1}\right)-z_{0}\left(z_{1}+z_{2}\right)\right] /\left(f z_{2}\right)}}
\end{aligned}
$$

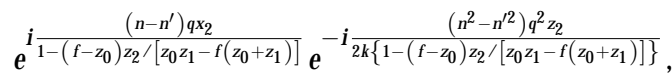

where ${ }^{*}$ means complex conjugated and $C_{3}$ has taken again all constants. Finally, we apply the finite size of the source and polychromatic light by performing two integrals over $x_{0}$ and $\lambda$ respectively,

$$
I_{F}\left(x_{2}, z_{2}\right)=\int_{-s / 2}^{s / 2} \int_{\lambda_{0}-\Delta \lambda / 2}^{\lambda_{0}+\Delta \lambda / 2} I_{2}\left(x_{2}, z_{2}\right) d \lambda d x_{0},
$$

where we have considered that the spectrum of the light source is homogeneous, with central wavelength $\lambda_{0}$ and width $\Delta \lambda$, 
and that the source size is $s$. For this spectrum the integral is easily solved,

$$
\begin{aligned}
& I_{F}\left(x_{2}, z_{2}\right)=C_{3} \sum_{n, n^{\prime}=-\infty}^{\infty} a_{n} a_{n^{\prime}}^{*} \\
& \operatorname{sinc}\left\{\frac{\left(n-n^{\prime}\right) q s}{2\left[-f / z_{2}+\left(-f+z_{1}+z_{2}\right) \triangle z /\left(f z_{2}\right)\right]}\right\} \\
& \times \operatorname{sinc}\left(\frac{\left(n^{2}-n^{\prime 2}\right) q^{2} z_{2} \triangle \lambda}{8 \pi\left\{1-z_{2} \triangle z /\left[f^{2}+\left(f-z_{1}\right) \triangle z\right]\right\}}\right) \text { 5) } \\
& e^{i \frac{\left(n-n^{\prime}\right) q x_{2}}{\left\{1-z_{2} \Delta z /\left[f^{2}+\left(f-z_{1}\right) \Delta z\right]\right\}}} e^{-i \frac{\left(n^{2}-n^{\prime 2}\right) q^{2} z_{2}}{2 k_{0}\left\{1-z_{2} \Delta z /\left[f^{2}+\left(f-z_{1}\right) \Delta z\right]\right\}}},
\end{aligned}
$$

where $\operatorname{sinc}(a)=\sin (a) / a, k_{0}=2 \pi / \lambda_{0}$ and $\Delta z=z_{0}-f$. As can be observed, the effects of the polychromaticity and finite size of the source are present into the sinc functions. The first sinc function corresponds to the effect of the source size and the second sinc function corresponds to the effect of the polychromaticity. These functions modulate the amplitude of the corresponding terms and the value of their arguments determines which one has dominant behavior, [26]. We show in Fig. 2 two examples of intensity computed with Eq. (5). They correspond to two different situations. The first one corresponds to a slightly divergent beam with a source of size $s=50 \mu \mathrm{m}$ and the second one corresponds to a slightly divergent beam with a source of size $s=600 \mu \mathrm{m}$, both with the same remaining parameters. As a general remark, we can observe that fringes disappear as we separate from the grating. This behavior is due to the finite size of the source. This fact has also been reported in [26], considering a finite extension polychromatic source propagating in free space. It is more clear in Fig. 2(b) where this effect is notorious due to the large size of the source. All these considerations make important to experimentally measure the fringes at the zero-th or first Talbot plane so that visibility of fringes is high enough to be accurately measured. In fact, the maximum distance with visible fringes is given by the sinc functions in Eq. (5). As a representative example, we show in Fig. 3 both sinc functions considering $n=1$ and $n^{\prime}=0$ for the same case as Fig. 2(b), where $t_{1}=\operatorname{sinc}\left\{q s / 2\left[-f / z_{2}+\left(-f+z_{1}+z_{2}\right) \Delta z /\left(f z_{2}\right)\right]\right\}$ and $t_{2}=$ $\operatorname{sinc}\left(q^{2} z_{2} \triangle \lambda / 8 \pi\left\{1-z_{2} \triangle z /\left[f^{2}+\left(f-z_{1}\right) \triangle z\right]\right\}\right)$. As can be observed, the polychromaticity of the source is not restrictive (dashed line) but the effect of the source size is notorious (solid line). From $t_{1}$ we can calculate the distance at which the contrast of the fringes decays to zero, resulting

$$
z^{\prime}=\left[\triangle z\left(f-z_{1}\right)+f^{2}\right] /[\triangle z-f q s / 2 \pi]
$$

In this particular case, the contrast of the fringes decays to zero for around $z^{\prime}=10 \mathrm{~mm}$. Anyway, we need to place the observation plane closer to the grating than $z^{\prime}$, in order to obtain fringes with enough visibility to be measured.

Once we have determined the distance of the observation plane to have high contrast fringes, the collimation method consists of measuring the period of the fringes for constant distances $z_{1}$ and $z_{2}$ and comparing it with the period of the grating, that we assume well known. Thus, the period of the fringes around Talbot planes, $d^{\prime}$, can be extracted from the first exponential factor of Eq. (5) resulting in

$$
d^{\prime}=d\left[1-\frac{z_{2} \triangle z}{f^{2}+\left(f-z_{1}\right) \triangle z}\right]
$$

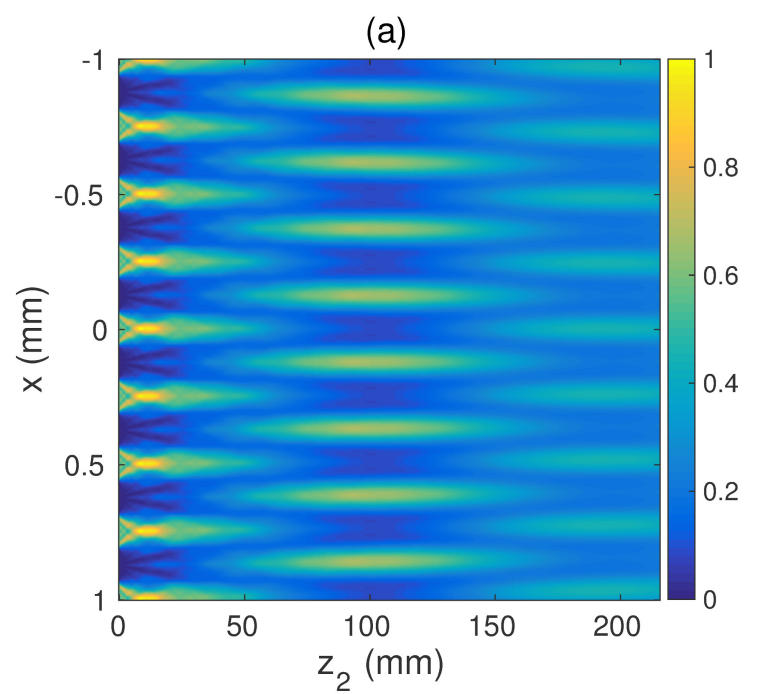

(b)

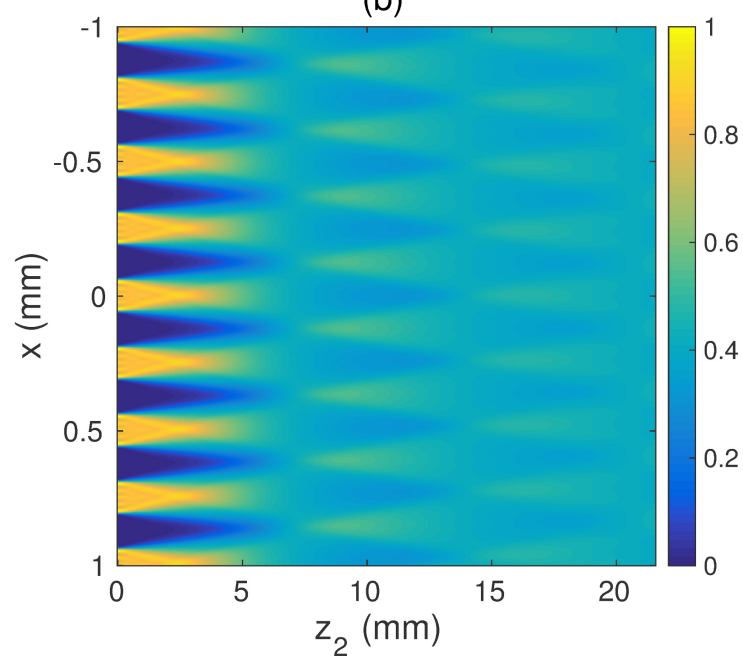

Fig. 2. Analytical near field intensity after the grating illuminated by a polychromatic finite extension light source passing through a lens obtained with Eq. (5). The used parameters are $\lambda_{0}=580 \mathrm{~nm}, \Delta \lambda=400 \mathrm{~nm}, d=250 \mu \mathrm{m}$, $f=25 \mathrm{~mm}, z_{1}=10 \mathrm{~mm}, n=n^{\prime}=(-9,-7, \ldots,-1,0,1, \ldots, 7,9)$, $\triangle z=0.1 \mathrm{~mm}$, and (a) $\mathrm{s}=50 \mu \mathrm{m}$, (b) $\mathrm{s}=600 \mu \mathrm{m}$.

This result is also obtained in [5]. From Eq. (7), the error in the positioning of the source with respect to the focal point of the collimating lens is given by

$$
\delta z=\frac{f^{2}}{\left(z_{1}-f\right)+\frac{d}{\delta d} z_{2}},
$$

where $\delta d=d-d^{\prime}$ is the error in the period measurement. For an experimental set-up with a fixed lens, known diffraction grating and fixed distance between lens and grating, the error in the positioning of the source decreases in terms of the distance to the observation plane, $z_{2}$. The uncertainty in the positioning of the lens decreases as we separate from the grating. Lamentably, the distance $z_{2}$ cannot be arbitrary chosen due to the finite size of the source that produces a decreasing of the contrast of the fringes. We have defined $z^{\prime}$ as the distance at which the contrast of the fringes decays to zero, Eq. (6). As a criterion, considering collimation situation, $\Delta z=0$, we can 


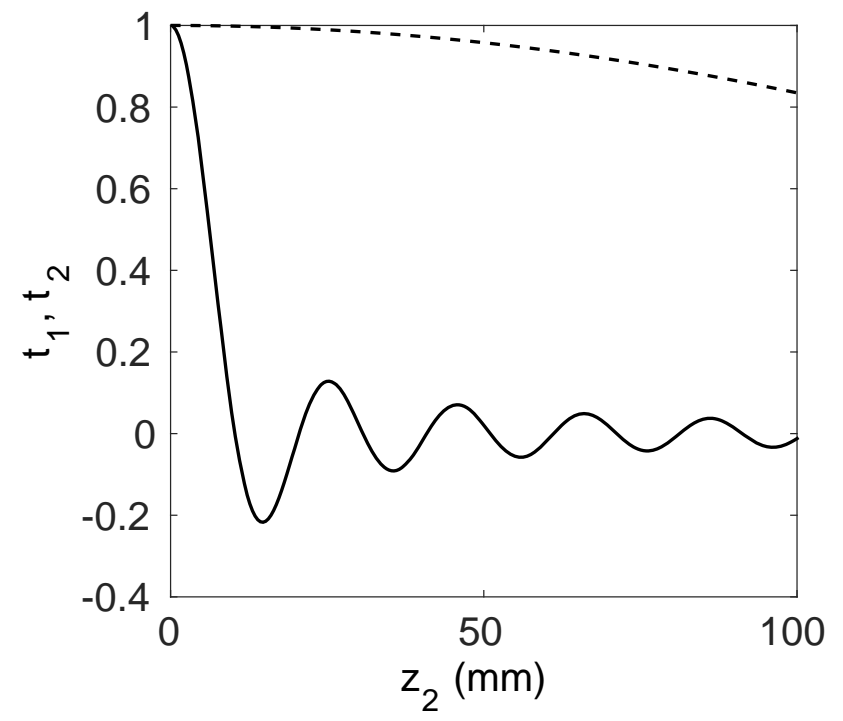

Fig. 3. Analytical sinc functions from Eq. (5) obtained for $n=$ 1 and $n^{\prime}=0 . t_{1}$ (solid line) corresponds to the effect of the finite size of the source and $t_{2}$ (dashed line) corresponds to the polychromaticity of the source. The used parameters are $\lambda_{0}=$ $580 \mathrm{~nm}, \triangle \lambda=400 \mathrm{~nm}, d=250 \mu \mathrm{m}, f=25 \mathrm{~mm}, z_{1}=10 \mathrm{~mm}$, $\triangle z=0.1 \mathrm{~mm}$, and $\mathrm{s}=600 \mu \mathrm{m}$.

establish $z_{2}^{\max }=z^{\prime} / 2$ as the maximum distance with fringes with measurable contrast. Then substituting this value of $z_{2}$ into Eq. (8), the error in the positioning of the source in terms of the source size, $s$, can be defined as

$$
\delta z=\frac{f^{2}}{z_{1}-f\left(1+\frac{d^{2}}{2 s \delta d}\right)},
$$

We show in Fig. 4(a) the behavior of the fringes with $f=25 \mathrm{~mm}$, $z_{1}=10 \mathrm{~mm}$, and $z_{2}=0.1 \mathrm{~mm}$. Each column of the figure corresponds to a vertical integration of the fringes for each $\Delta z$. Besides we plot in Fig. 4(b) the dependence of the period on $\triangle z$ following Eq. (7). As it was expected, the period of the fringes is equal to the period of the grating for $\triangle z=0 \mathrm{~mm}$.

\section{EXPERIMENTAL RESULTS}

To corroborate the analytical results, we have performed an experiment consisting of collimating a high power white LED of size $s=0.6 \mathrm{~mm}$ by means of a lens. LEDs are not usually circularly symmetric and therefore, we need to choose one direction for collimation. We have chosen a collimating lens of focal length $f=25 \mathrm{~mm}$ and a Ronchi diffraction grating of period $d=250 \mu \mathrm{m}$. The camera used to acquire the fringes is a UI-164xLE-C model by IDS, whose pixel size is $3.6 \mu \mathrm{m} \times 3.6 \mu \mathrm{m}$. Considering a central wavelength of $\lambda_{0}=$ $580 \mathrm{~nm}$, the corresponding Talbot distance is $z_{T}=2 d^{2} / \lambda \simeq$ $215.52 \mathrm{~mm}$. Besides, considering collimation state in Eq. (6), $\Delta z=0, z^{\prime} \simeq 10,41 \mathrm{~mm}$ and $z_{2}^{\max } \simeq 5.205 \mathrm{~mm}$. Therefore, we need to mesure the fringes in the zero-th order self-image or "shadow" of the grating. We place the camera as close to the grating as possible to assure high contrast of the fringes. The experiment consists of displacing the source around the focal plane of the collimating lens and acquiring the fringes by using the CMOS camera. The movement is performed by (a)

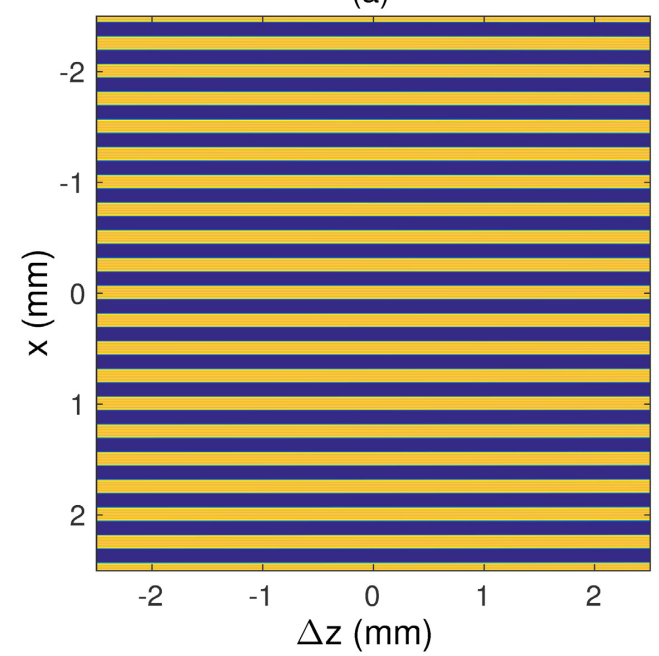

(b)

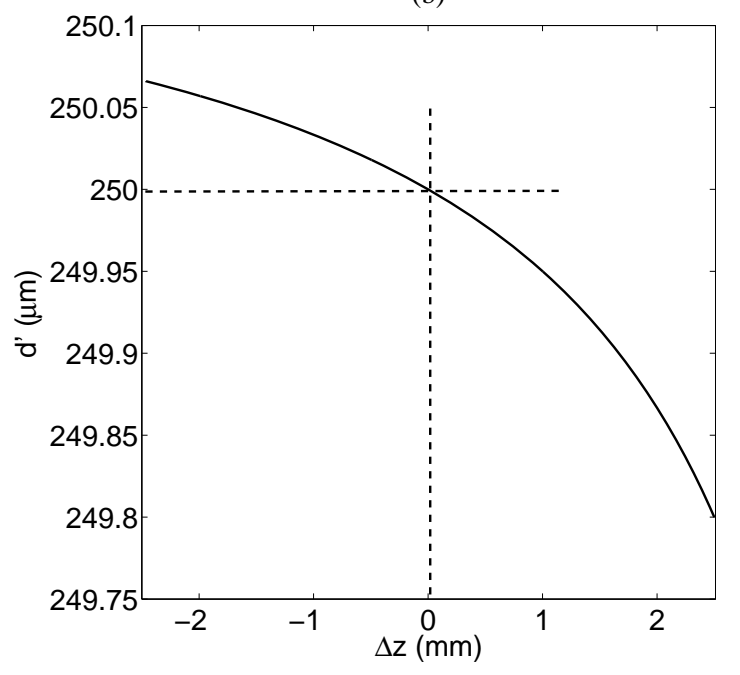

Fig. 4. (a) Analytical fringes with $d=250 \mu \mathrm{m}, f=25 \mathrm{~mm}$, $z_{1}=10 \mathrm{~mm}, z_{2}=0.1 \mathrm{~mm}$ and $s=600 \mu \mathrm{m}$ and (b) period of the fringes shown in Fig. 4(a).

using a motorized linear stage model M-521 by PI. We assure the measurements free from vibrations and so on by pausing the movement between every two measurements for a while. We show in Fig. 5 the experimental fringes obtained in terms of $\triangle z$. Each column in Fig. 5 corresponds to a vertical integration parallel to the fringes acquired with the camera. After that, we calculate the period of the fringes in the same fashion as [21]. We use the variogram function which allows reducing noise and filtering inhomogeneities of the illumination source. As it is shown in Fig. 6(a), the same curvature to that obtained analytically appears experimentally. We have fitted the data to Eq. (7), calculating also the confidence intervals to $95 \%$. The coefficient of determination for the fitting is $R^{2}=0.999$, showing an excellent fitting of the experimental data to the analytical equation. According to Eq. (7), the parameters obtained from the fitting are: $z_{1}=6.456 \pm 0.132 \mathrm{~mm}, z_{2}=425 \pm$ $33 \mu \mathrm{m}$. The residuals are shown in Fig. 6(b). The error in the period estimation for the grating of period $d=250 \mu \mathrm{m}$ has been determined experimentally from Fig. 6, resulting $\delta d=17.2 \mathrm{~nm}$ at $\Delta z=0$. Substituting all values into Eq. 8 , the theoretical error 


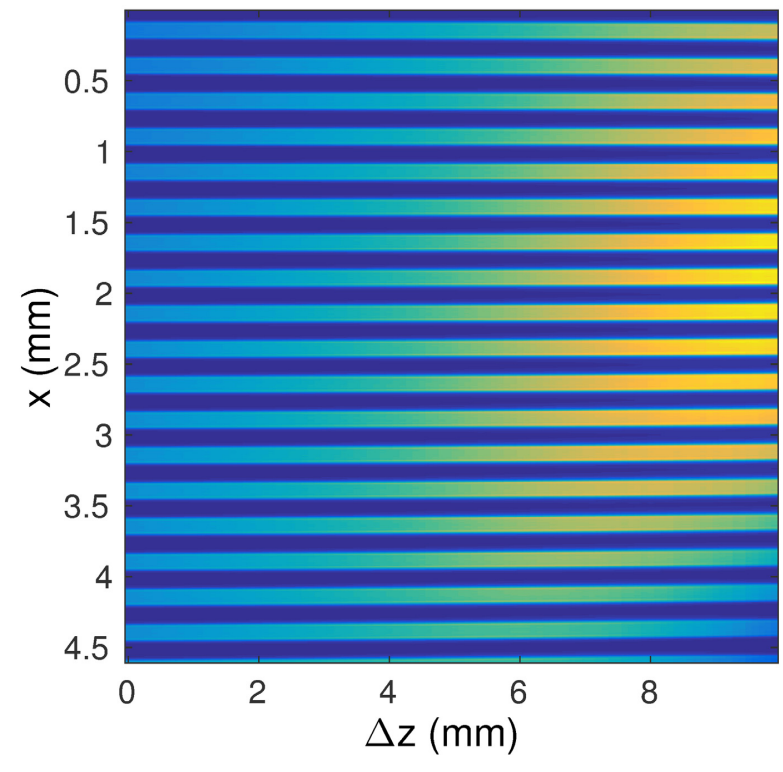

Fig. 5. Experimental fringes obtained for a grating of period $d=250 \mu \mathrm{m}$ using a white LED of size $s=0.6 \mathrm{~mm}$ and a collimating lens of focal length $f=25 \mathrm{~mm}$. The different colors are due to inhomogeneities of the beam but do not influence the period measurements.

in the positioning of the source results $\delta z=101.42 \mu \mathrm{m}$. From the experimental data and the fitting we can predict the position in which the beam results collimated. For this particular experiment, the location is $\Delta z+\alpha=1148.93 \mu \mathrm{m}$, being $\alpha$ an unknown parameter depending on the initial position of the motorized stage. It corresponds to the position that fulfills $d^{\prime}=d$. We may suppose the fitting as linear around $d^{\prime}=d$, see zoom in Fig. 6(a), and therefore the error in positioning the source at the focal point of the lens can be experimentally obtained from Fig. 6(a) resulting in $\delta z_{\exp }=92 \mu \mathrm{m}$. This value is estimated by measuring the horizontal distance from the fitted curve to the confidence interval. As can be observed, it results of the same order than the theoretical prediction.

\section{CONCLUSIONS}

Many accurate collimation techniques have been developed for collimating monochromatic point light sources. In this work, we present a collimation technique and testing valid for polychromatic finite extension light sources based on comparing the period of the fringes propagated from the grating and the period of the proper grating. Analytical equations are obtained to predict the error in the positioning of the source in terms of the source size and set-up parameters. Besides, experimental results that corroborate the analytical approach are obtained when the light source is a white LED of size $s=0.6 \mathrm{~mm}$. The achieved experimental accuracy in the positioning of the source with respect to the focal plane of the lens results $\delta z_{\text {exp }}=92 \mu \mathrm{m}$. This work is highly useful in applications where finite size polychromatic sources need to be collimated accurately.

\section{ACKNOWLEDGMENTS}

This work was started while Francisco José Torcal-Milla was contracted by the Universidad Complutense de Madrid. (a)

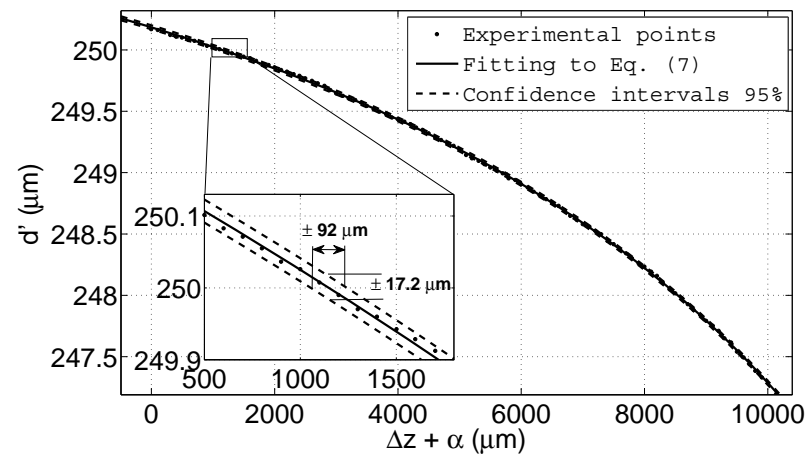

(b)

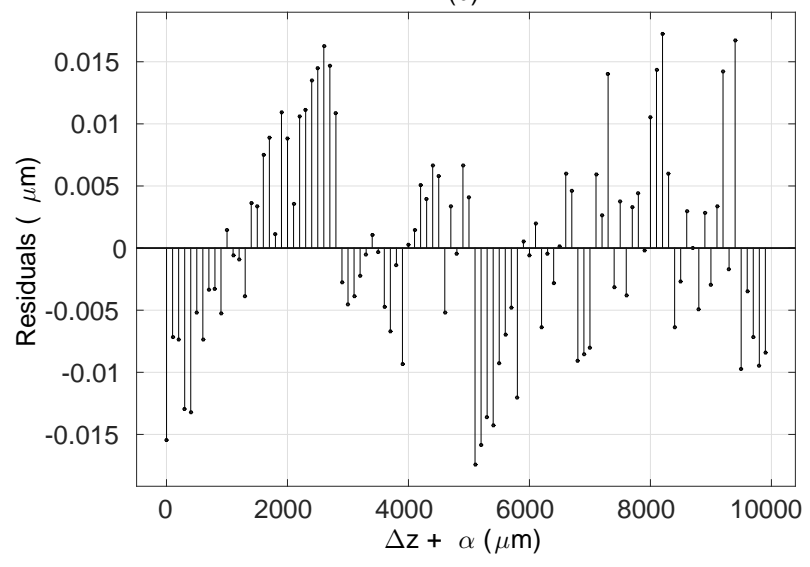

Fig. 6. (a) Period of the experimental fringes of Fig. 5 in terms of $\Delta z$, experimental data (dots), fitting (solid line), confidence intervals (dashed lines) and (b) residuals of the fitting. $\alpha$ is an unknown constant dependent on the initial position of the linear stage used to displace the source.

This work has been supported by project SPIP2015-01812 "Dispositivo LiFi de comunicación de datos sensibles de vehículos para incremento de la seguridad vial" of the Ministerio de Interior of Spain.

\section{REFERENCES}

1. D. E. Silva. A simple interferometric method of beam collimation. Appl. Opt., 10(8):1980_1-1982, 1971.

2. MVRK Murty. The use of a single plane parallel plate as a lateral shearing interferometer with a visible gas laser source. Appl. Opt., 3(4):531-534, 1964.

3. D Joyeux and Yael Cohen-Sabban. High magnification self-imaging. Appl. Opt., 21(4):625-627, 1982.

4. Mahendra $P$ Kothiyal and Rajpal S Sirohi. Improved collimation testing using talbot interferometry. Appl. Opt., 26(19):4056-4057, 1987.

5. Krzysztof Patorski. The self-imaging phenomenon and its applications. Prog. Optics, 27:1-108, 1989.

6. AR Ganesan and Putcha Venkateswarlu. Laser beam collimation using talbot interferometry. Appl. Opt., 32(16):2918-2920, 1993.

7. J Choi, Gerard M Perera, MD Aggarwal, RP Shukla, and MV Mantravadi. Wedge-plate shearing interferometers for collimation testing: use of a moiré technique. Appl. Opt., 34(19):3628-3638, 1995.

8. JS Darlin, MP Kothiyal, and RS Sirohi. A phase-conjugate twyman-green interferometer with increased sensitivity for laser beam collimation. J. Mod. Opt., 45(11):2371-2378, 1998.

9. D. Malacara. Optical Shop Testing. Wiley Series in Pure and Applied Optics. Wiley, 2007.

10. Shashi Prakash, Santosh Rana, Satya Prakash, and Osami Sasaki. 
Automated collimation testing using a temporal phase shifting technique in talbot interferometry. Appl. Opt., 47(31):5938-5943, 2008.

11. S Yokozeki, K Patorski, and K Ohnishi. Collimation method using fourier imaging and moiré techniques. Opt. Commun., 14(4):401-405, 1975.

12. KV Sriram, Mahendra P Kothiyal, and Rajpal S Sirohi. Self-referencing collimation testing techniques. Optical Engineering, 32(1):94-100, 1993.

13. Sarayut Deachapunya and Sorakrai Srisuphaphon. Sensitivity of transverse shift inside a double-grating talbot interferometer. Measurement, 58:1-5, 2014.

14. Sorakrai Srisuphaphon and Sarayut Deachapunya. The study of wave motion in the talbot interferometer with a lens. Wave motion, 56:199204, 2015.

15. Chandra Shakher, Shashi Prakash, Daya Nand, and Rajesh Kumar. Collimation testing with circular gratings. Appl. Opt., 40(8):1175-1179, 2001.

16. Lei Huang and Xianyu Su. Method for acquiring the characteristic parameter of the dual-spiral moiré fringes. Opt. Lett., 33(8):872-874, 2008.

17. Krzysztof Patorski, Krzysztof Pokorski, and Maciej Trusiak. Circularlinear grating talbot interferometry with moiré fresnel imaging for beam collimation. Opt. Lett., 39(2):291-294, 2014.

18. Luis Miguel Sanchez-Brea, Francisco Jose Torcal-Milla, Francisco Javier Salgado-Remacha, Tomás Morlanes, Isidoro JimenezCastillo, and Eusebio Bernabeu. Collimation method using a double grating system. Appl. Opt., 49(17):3363-3368, 2010.

19. Francisco Jose Torcal-Milla, Luis Miguel Sanchez-Brea, and Jose Maria Herrera-Fernandez. Lissajous figure-based single-frame collimation technique. Sensors and Actuators A: Physical, 233:259266, 2015.

20. Xiaoyu Chen, Jinbo Su, Xiangqun Cao, Bin Lin, and Bo Yuan. Research on the moire fringes formed by circular and linear grating. Chinese Optics Letters, 9(s1):10702, 2011.

21. Luis Miguel Sanchez-Brea, Francisco Jose Torcal-Milla, Jose Maria Herrera-Fernandez, Tomas Morlanes, and Eusebio Bernabeu. Selfimaging technique for beam collimation. Opt. Lett., 39(19):5764-5767, 2014.

22. Henry Fox Talbot. Lxxvi. facts relating to optical science. no. iv. The London and Edinburgh Philosophical Magazine and Journal of Science, 9(56):401-407, 1836.

23. Reena Disawal, Jitendra Dhanotia, and Shashi Prakash. Improved measurement characteristics in collimation testing using lau interferometry and fourier fringe analysis technique. Precision Engineering, 38(4):948-955, 2014.

24. Santosh Rana and Shashi Prakash. Collimation testing by use of the lau effect coupled with moiré readout. Applied optics, 45(14):32133217, 2006.

25. KV Avudainayagam and S Chitralekha. Lau effect and beam collimation. journal of modern optics, 44(1):175-178, 1997.

26. Francisco Jose Torcal-Milla and Luis Miguel Sanchez-Brea. Achromatic self-imaging with finite extension light sources. Journal of Optics, 17(12):125605, 2015. 